CARNETS OE Carnets de géographes

GÉOGRAPHES.

4 | 2012

Géographies critiques

\title{
Pour une géographie sociale de l'action
}

\section{Nicolas Bautes et Clement Marie dit Chirot}

\section{(2) OpenEdition}

\section{Journals}

Édition électronique

URL : http://journals.openedition.org/cdg/982

DOI : $10.4000 /$ cdg.982

ISSN : 2107-7266

Éditeur

UMR 245 - CESSMA

\section{Référence électronique}

Nicolas Bautes et Clement Marie dit Chirot, «Pour une géographie sociale de l'action », Carnets de géographes [En ligne], 4 | 2012, mis en ligne le 01 septembre 2012, consulté le 30 avril 2019. URL: http://journals.openedition.org/cdg/982 ; DOI : 10.4000/cdg.982

\section{c) (i) $९$}

La revue Carnets de géographes est mise à disposition selon les termes de la Licence Creative Commons Attribution - Pas d'Utilisation Commerciale - Pas de Modification 4.0 International. 


\title{
POUR UNE GÉOGRAPHIE SOCIALE DE L'ACTION
}

\author{
NICOLAS BAUTES \\ Maître de conférence en géographie \\ Université de Caen \\ UMR ESO/CEIAS \\ nicolas.bautes@unicaen.fr \\ CLEMENT MARIE DIT CHIROT \\ Doctorant en géographie \\ Université de Caen \\ UMR ESO \\ clement.marie-ditchirot@unicaen.fr
}

\section{Résumé}

Partant de débats actuels sur le retour de la pensée critique dans les sciences sociales, cet article propose d'interroger, à partir de choix théoriques et méthodologiques, et de présupposés éthiques et déontologiques, la possibilité d'une géographie sociale de l'action. Le rapport particulier au terrain qui caractérise, depuis ses débuts, l'émergence de la géographie sociale est ici envisagé comme un élément indissociable de la formulation d'une pensée critique, en prise avec la société. Ce questionnement prolonge des réflexions déjà posées par des auteurs issus de disciplines connexes (sociologie, anthropologie) et lie la portée critique du discours scientifique à sa diffusion dans la société. Ce postulat nous amène à envisager le rapport entre théorie et pratique, et à poser les conditions d'une géographie de l'action plaçant l'efficacité des savoirs critiques au cœur du projet scientifique.

\section{Introduction $^{1}$}

La posture des géographes vis-à-vis de leur objet - avant l'espace, la société ${ }^{2}$ - s'appuie sur des choix individuels, associés le cas échéant à des orientations politiques voire à

\footnotetext{
${ }^{1}$ Les auteurs souhaitent remercier Robert Hérin pour sa disponibilité et son ouverture à l'échange auquel nous l'avons invité, qui s'est notamment traduit par la tenue d'un entretien compréhensif dont certains extraits ont été mobilisés dans le cours du texte.

${ }^{2}$ Nous faisons ici référence à la vive prise de position de R. Rochefort in « Réflexions liminaires sur la géographie sociale ", Actes du Colloque de Lyon, 14-16 oct. 1982, éd. par D. Noin, 1983.
} 
des idéologies qui gagnent, pour être mis en récit, discutés et diffusés en vue de nourrir un discours scientifique réflexif, à être explicités ${ }^{3}$. Or, les travaux de géographes tant écrits que présentés oralement font souvent l'économie de ces préalables qui semblent pourtant inévitables pour transmettre des points de vue, débattre, ou défendre des idées. Ceci est d'autant plus étonnant que ces précautions figurent au cœur des préoccupations de disciplines connexes comme l'anthropologie ou la sociologie, desquelles la géographie humaine s'est beaucoup nourrie ${ }^{4}$. Comment expliquer cet état de fait, alors même que la géographie française est traversée, depuis les années 1970, par ces champs d'interrogation, notamment impulsés par des auteurs engagés ayant contribué à prôner une géographie "de son temps", capable de contribuer à "la prise de conscience collective et à la solution des problèmes de société » (Rochefort, 1983) ?

Si le contexte actuel de la production du savoir scientifique marqué, en sciences sociales comme pour dans d'autres champs de la recherche, par des impératifs de productivité - dans leur volet professionnalisant ou dans le domaine de l'aide à la décision notamment, mais pas uniquement -, peut en partie expliquer cette économie, il ne permet cependant pas de saisir le mouvement qui conduit de nombreux auteurs à combiner, dans leur pratique professionnelle, une attitude qui vise à répondre à l'injonction à l'adage " publier ou périr " (publish or perish), et dans le même temps à revendiquer des formes d'engagement adossées à un positionnement critique. Cette question, qui dépasse le cadre de la seule géographie, renvoie à la place de la critique dans le discours scientifique et, plus largement, à la portée de cette critique, qu'elle soit prise de position ou contestation.

De notre point de vue, une trop faible lumière permet d'éclairer tant les postures c'est-à-dire les rapports des chercheur-es au terrain - les emprunts théoriques et méthodologiques, que les processus dans lesquels elles s'inscrivent: le mouvement apparemment contradictoire entre prise de position critique - souvent contre les réformes et le changement de désignation du chercheur en " produisant » - et discours critique ou engagé.

\footnotetext{
${ }^{3}$ Notre propos général est construit autour d'une prise de parti, et donc un parti pris, vis-à-vis de la conjoncture actuelle à laquelle est soumis le monde de la recherche. Il nous semble en effet que ce contexte, duquel participe l'injonction à une production scientifique "d'excellence ", influe sur la production scientifique en détournant une partie du travail de chercheur de préoccupations qui, selon nous, fondent la légitimité du discours académique : le questionnement sur le rapport à l'Autre, duquel nous puisons la matière qui construit notre regard sur les sociétés, et plus largement la contribution des chercheur(e)s au monde social dans lequel ils s'insèrent. Au delà de cette posture, partiellement biaisée par notre condition de chercheurs (marquée ici par notre genre, notre position sociale à l'intérieur et en dehors du champ académique et, le cas échéant, notre origine géographique), ce discours est construit à partir de nos propres expériences et de nos lectures, et n'est en aucun cas le fait d'un quelconque parti politique. Les deux auteurs, dont les statuts dans le monde académique diffèrent à ce jour, ont été ou sont tous deux marqués, comme de nombreux autres, par les difficultés inhérentes à accéder à une fonction permanente et non précaire au sein du monde académique.

${ }^{4}$ Parmi de nombreux travaux ayant contribué à clarifier les apports de ces disciplines à la géographie, on peut citer ici la contribution de J. Poirier (1967) écrite dans une période marquée par de vifs débats autour du renouvellement de la géographie : "Géographie humaine, ethnologie et sociologie ", Annales de l'Université de Madagascar - Lettres, volume 6, 1967 pp. 125-138. (En ligne: madarevues.recherches.gov.mg/revues/pdfxfiles/anal-lettres6(6).pdf), Page consultée le 27 avril 2012.
} 
Cette contribution s'organise autour de quatre axes. Le premier envisage un bref retour sur la question de l'engagement du chercheur et, plus largement, de sa place parmi les enquêtés et de son rôle social tout au long de l'épreuve du terrain, dont $M$. de la Soudière mentionne qu'il convient d'en " démystifier certaines fausses évidences ou idées reçues (...), en montrant en particulier comment son privilège d'observateur de la vie sociale se transforme alors pour lui en cadeau empoisonné » (De la Soudière, 1998, p.94). Partant de courts extraits d'expériences de recherche, le second aborde ce champ d'interrogations en questionnant nos propres postures de géographes, à la fois sur le terrain et dans la démarche de production - écrite et parlée, mais aussi enseignée ${ }^{5}$. Chacun de ces extraits fait référence à des situations qui ont traversé et transformé notre expérience d'enquêteur, et qui nous ont conduits à nous questionner, à douter et à prendre position, à prendre parti ou à être pris à parti. Ces moments particuliers, durant lesquels les logiques du champ académique interfèrent avec la pratique sociale des acteurs, nous ont parfois permis de mesurer les enjeux de notre présence, mais aussi de relativiser la portée de notre discours, envisagé notamment dans sa forme finale, à savoir la production écrite. C'est à la lumière de ces expériences que nous avons souhaité interroger notre rapport à l'engagement, fil conducteur de cette contribution, dont le troisième moment, partant du constat de la portée limitée du discours académique, invite à explorer plus avant les questions d'éthique vis-à-à-vis du terrain et, avec elles, les fondements d'une science sociale critique. Enfin, cette discussion se clôt sur les possibles perspectives qui s'offrent aux chercheurs pour s'engager dans la production d'un savoir universitaire critique ${ }^{6}$ qui soit "socialement utile" (Hérin, 1984, p.70; Collignon, 2001, p.132) notamment au regard d'expériences locales de coproduction des savoirs impliquant chercheurs et non-chercheurs.

\section{Premiers jalons pour questionner la production d'un savoir critique en géographie : la question de l'engagement du chercheur}

En tant que chercheurs en sciences sociales, géographes de formation travaillant dans des sociétés du Sud ${ }^{7}$ marquées par de vives inégalités sociales observées dans le

\footnotetext{
${ }^{5}$ Même si cet aspect n'est pas approfondi dans cette contribution, il nous semble important de le mentionner, en vue d'envisager des réflexions futures.

${ }^{6}$ Le terme "critique " est ici entendu dans un sens plus large que celui qui est mentionné par B. Collignon. Notre acception inclut donc à la fois la géographie "critique " anglo-saxonne des années 1990, « l'ensemble des géographies contestataires ayant développé (...) des travaux de déconstruction des discours, sous l'influence des théories postmodernes ", la géographie radicale anglo-saxonne, " plus théorique et moins soucieuse d'engagement concret » (Collignon, 2001 : 294), et la géographie sociale, même si sous cette appellation ne figure par l'ensemble des travaux concernés par la question de la critique, de la contestation et de l'engagement.

${ }^{7}$ Le "Sud " ne constitue pas dans notre propos un référent géographique renvoyant à une quelconque forme d'exotisme ou à " une altérité confortablement confinée spatialement » (Hancock, $2007: 1)$. Si nous sommes conscients du fait que les discours contribuent à reproduire ce qu'ils décrivent, cette catégorie d'analyse nous apparaît néanmoins aujourd'hui encore féconde pour penser un certain nombre d'asymétries et d'inégalités à l'échelle du Monde, leur construction à l'échelle du temps long de l'Histoire, mais aussi leur traduction à des échelles d'analyse plus fines. De ce point de vue, nous rejoignons la position du sociologue Boaventura de Sousa Santos et son invitation à une " épistémologie du Sud ", un regard construit à partir d'un "Sud " irréductible à sa dimension spatiale mais envisagé comme une « métaphore de la souffrance humaine causée systématiquement par le colonialisme et le
} 
quotidien de nos terrains de recherche, mais aussi en tant que personnes, nos expériences nous ont conduits non seulement à être témoins et, parfois, à être contraints de nous positionner face à des situations que nous jugions intolérables: discriminations, conflits, voire violences tout à la fois physiques, sociales et symboliques. L'enjeu de cette situation est justement décrit par Morelle et Ripoll (2009) qui soulignent que " sur le terrain, la question de la justice et de l'injustice, et plus largement de "l'engagement ", n'est plus seulement un problème théorique et abstrait. Elle se pose et même s'impose pratiquement, dans des situations vécues directement par les chercheur-es, auxquelles ils sont personnellement confrontés ici et maintenant, auxquelles ils doivent réagir d'une manière ou d'une autre. " (Ripoll \& Morelle, 2009, p.159).

Il semble dès lors important de questionner ces réactions qui prennent part au premier chef à une volonté de nous interroger sur notre position dans le monde social qui nous entoure et sur les exigences de cette position, que J-B Racine, dans un compte-rendu de lecture d'un ouvrage de J-P Ferrier, décrivait " en termes de contraintes d'équilibre, d'autonomie et d'autocréation " (Racine, 1989, p.52). Aussi, la " "mise en science" de l'expérience irremplaçable du rapport au territoire » (p.53) que constitue le terrain du géographe ne peut se faire sans éclaircir la nature et la portée de l'engagement.

Lors de nos enquêtes en Inde, au Mexique et au Brésil, nous avons tour à tour pris parti, parlé publiquement, nous sommes tus dès lors que notre parole était susceptible de menacer l'un de nos " informateurs " ou qu'elle pouvait nuire à notre entreprise de recherche, la fameuse "connaissance scientifique». Nous avons aussi, à plusieurs reprises, pris part, à des expressions contestataires, à l'émergence de mobilisations, occupant des rôles très divers dans ces mouvements. Cet engagement nous apparaissant sinon évident, important, fort de convictions parfois inavouées - ou du moins pas toujours explicitées -, nous nous sommes le plus souvent placés du côté de ceux qui nous apparaissaient comme les dominés ou, pour reprendre la désignation proposée par G. Spivak, les subalternes ${ }^{8}$. Cette position ne va cependant pas de soi : sans jamais apparaitre comme un présupposé, elle a, chaque fois, été guidée par le contexte de l'enquête qui, fondée sur des entretiens compréhensifs, ont mis en évidence des situations de domination, des ressources différenciées selon les acteurs interrogés et, dans plusieurs cas à l'étude, des situations de souffrance liées à cette domination. Notre position a été choisie non comme un parti pris initial, mais révélée par la parole, les gestes et plus généralement les récits de nos interlocuteurs.

Le choix d'une telle position n'est certes pas sans poser problème, tant " les catégories de représentation et d'action sur la société sont loin d'être immanentes et qu'elles font l'objet d'un travail d'élaboration souvent conflictuel où les capacités d'intervention sont inégalement réparties entre catégorisant et catégorisés" (Martiniello \& Simon, 2005, p.7). Nous l'envisageons ici comme un résultat, non celui de notre enquête proprement dite, mais comme le fruit d'un travail d'interprétation.

Le fait est que cette posture s'est peu à peu imposée, élaborée à partir du souci d'être au plus près de nos positions personnelles - à la fois dans le registre sensible et

capitalisme " (Santos, 2009: 12), Ceci, d'autant plus dans le contexte de pays dont l'influence économique et politique tend de plus en plus à s'affirmer dans le monde

${ }^{8}$ Spivak, G. (2006) Les subalternes peuvent-elles parler? Paris, Amsterdam, $110 \mathrm{p}$. 
politique -, et au travers de l'élaboration d'une méthodologie suffisamment structurée pour limiter les biais relatifs à la prise de parti univoque, et adoptée de manière à laisser nos interlocuteurs exprimer aussi librement que possible leurs réalités vécues. Face à cette parole, nous nous sommes placés comme les "'dépositaires' des récits recueillis " (Rochefort (1962), citée par Alduy \& Labussière, 2011, p.10) impliqués dans « un mode relationnel complexe qui évolue en fonction de paramètres théoriques et épistémologiques, mais aussi de la pratique du terrain elle-même " (/bid.).

II nous semble important d'aller plus avant dans cette réflexion sur le rôle social du chercheur en assumant notre engagement, entendu, après B. Verhaegen - au sens plein du terme -. Selon lui, l'engagement ne constitue pas « un obstacle à la validité de la connaissance ni à la rigueur de la démarche scientifique " mais "doit être recherché systématiquement et doit être assumé consciemment, car l'engagement volontaire et conscient du chercheur - et de son œuvre scientifique - (...) est une nécessité épistémologique. II est la condition fondamentale d'une connaissance adéquate, une condition de la validité de la connaissance, à la rigueur de la démarche scientifique ". (Verhaegen, cité par Omasombo, 1993 p.216).

Cette considération met en évidence la complexité inhérente aux liens entretenus par le chercheur à la fois avec le contexte idéologique dans lequel est inscrit son objet de recherche.

En enquêtant sur la spéculation foncière et immobilière dans une ville touristique mexicaine, nous avons approché les différentes parties impliquées dans des rapports sociaux complexes, allant de la connivence à la confrontation violente: pouvoirs publics, propriétaires fonciers, investisseurs locaux ou étrangers, mais aussi des groupes d'habitants mobilisés pour l'accès au logement et la défense de leur quartier, si durement construit grâce à des années de lutte sociale, face aux politiques de revalorisation économique menées conjointement par les pouvoirs publics et I'initiative privée.

Dans la même logique, mais dans d'autres contextes, à Rio de Janeiro au Brésil, à Udaipur (Rajasthan) ou à Mumbai en Inde, notre travail de recherche a nécessité de tisser des liens, parfois proches, avec des habitants contraints, dans le cadre de politiques de rénovation urbaine, d'être délogés; ou encore avec des acteurs engagés dans des activités illicites, voire criminelles; à être témoins de situations de corruption ou d'intimidation. Dans tous ces cas, les conflits observés sur le terrain devenaient des manifestations locales de contradictions existant au sein d'un système économique, politique et social plus large qu'il s'agissait de décrire, d'analyser et de critiquer, souvent, à partir d'une nécessaire mise à distance.

À ce sujet, l'ethnologue américain Jim Thomas estime que le chercheur en sciences sociales a l' " obligation éthique " d'être attentif au contexte idéologique dans lequel il est situé et qui le conditionne insidieusement. Nous avons pris la mesure de cette assertion alors qu'au cours de nos enquêtes nous avons pris la mesure de ce contexte, non pas traduit, à Rio de Janeiro ou à Mumbai, par les uniques signes lancés par des instances municipales observant une attitude répressive vis-à-vis des habitants de bidonvilles, mais également par l'existence d'autres formes d'exercices du pouvoir particulièrement assurés par des narcotrafiquants n'hésitant pas eux-mêmes à faire l'usage de la persuasion et de la force pour récompenser ou punir des habitants ayant trop parlé, ou ayant au contraire facilité l'activité - économique - de ces acteurs criminels. Lorsque l'enquête conduit à révéler de telles pratiques, c'est à la fois une 
partie importante du fonctionnement du système social qui peut être entrevue et la position du chercheur qui se trouve questionnée, notamment dans sa dimension éthique. Dans une telle configuration, jusqu'où aller pour expliquer?

S'inscrivant dans ce registre de réflexion, J. Thomas (1993, cité par Ghasarian, op.cit.) propose une ethnologie critique "qui ne se contenterait pas de décrire les choses, mais questionnerait ce rôle dans la production du savoir » étudiant la culture non plus seulement pour la décrire (" ce qui est », écrit Ghasarian) " mais pour la changer (ce qui devrait être), le but étant de faire entendre la voix des sujets (...) (plaçant) la recherche dans le domaine de la prescription plutôt que dans celui de la description " (Ibid.). Si l'invitation est tentante, il semble également important de noter, suivant C. Ghasarian, que " même si les implications éthiques de son travail ne doivent pas être négligées, il s'agit, pour celui-ci, de définir son rôle et de savoir quelles en sont les limites » (Ghasarian, 1997, p.191).

Relayant ces préoccupations, des travaux récents ont insisté sur les problèmes d'éthique rencontrés par les géographes dans le cadre de leurs recherches de terrain (Collignon, 2010; Morelle et Ripoll, 2009). Engagés dans leur fonction de chercheur(e)s, mais aussi en tant que personnes, dans un ensemble de relations avec d'autres acteurs - qu'il s'agisse d'individus, de groupes ou d'institutions, ceux-ci sont confrontés à différents types d'épreuves éthiques pouvant conduire à réinterroger une posture de recherche, particulièrement lorsque celle-ci affirme la volonté de produire une pensée distanciée et critique. Nos relations au terrain se sont ainsi tissées sur la base de tels cheminements et observations, construites à partir des échanges, prises de position et débats, plus largement autour de regards croisés engageant une nécessaire et parfois fragile relation de confiance, que nos démarches de terrain nous ont conduits à interroger. Une confiance qui nécessite, à ce stade de l'analyse, d'être mise en question, notamment parce qu'elle tend à confronter la parole de l'enquêté et la production d'un savoir dont la diffusion est souvent, pour des raisons que nous développerons, limitée à la fois dans ses canaux de transmission et dans sa capacité à générer un changement.

\section{La portée limitée du discours académique comme outil de changement social}

Les barrières rencontrées lors de multiples contacts avec des acteurs " dominants" (propriétaires fonciers, responsables des politiques urbaines à l'échelle municipale) sur nos terrains de recherche respectifs ont été à la hauteur de l'ouverture dont ont souvent fait preuve, à notre égard, les collectifs d'habitants, les représentants $d$ 'associations ou les leadeurs syndicaux engagés dans des conflits avec les institutions étatiques et de leur contribution active à nos démarches de recherche respectives. Avant d'obtenir des documents officiels sur les politiques et projets urbains et de pouvoir réaliser des entretiens avec des cadres des services d'urbanisme locaux, il aura fallu passer plusieurs filtres et montrer "patte blanche ": à plusieurs reprises, nous avons été conduits à rassurer nos interlocuteurs en précisant notre fonction, mais surtout les usages que nous ferions des résultats de nos entretiens. Au Mexique, il a fallu insister sur le fait que nous n'étions pas journalistes, mais chercheurs en géographie et étrangers aux jeux sociaux locaux, et que si les résultats de notre recherche étaient publiés, ils le seraient dans des revues scientifiques et non dans la presse. Convaincus du caractère inoffensif de notre démarche, certains de ces 
interlocuteurs appartenant au champ du pouvoir politique ou économique ont, parfois, consenti à s'ouvrir à notre quête d'informations. À Mumbai, dans le contexte du projet de réhabilitation d'un bidonville situé au cœur des enjeux fonciers métropolitains (Behrampada, voir Bautès et ali., 2011), la rencontre avec les responsables de groupes immobiliers et avec les représentants d'une association d'habitants, tous partie prenante d'une procédure de relogement de familles encadrée par l'État s'est, à plusieurs reprises, avérée délicate : face à des transactions souvent informelles, relevant d'arrangements ou de contrats tacites, la présence d'un chercheur - étranger de surcroit - peut être d'autant plus dérangeante que ni la nature de son enquête ni la portée de son discours ne semblent être aisément éclairés. Dans une favela de Rio de Janeiro, au détour d'une rue, accompagné par l'un de nos interlocuteurs, nous croisons un groupe de jeunes hommes qui, à notre passage, prononcent le nom du reporter séquestré puis assassiné en 2002 lors d'une investigation pour la TV Globo dans une favela de la ville: "tiens, en voilà un qui se prend pour Tim Lopes. Je serais attentif, hein, à sa place !».

À l'inverse, après un entretien avec des membres d'une association d'habitants d'un quartier populaire de la ville touristique de Playa del Carmen, au Mexique, les personnes interrogées ont souhaité aborder l'usage futur des informations recueillies dans le cadre de notre recherche. "Qu'est-ce que vous comptez faire de toutes ces informations?" ". Nous expliquions alors qu'en plus d'une thèse, nous écririons probablement un article et tenterions de le faire publier dans une revue. "Ah... Dans la revue Proceso ? ". Enthousiaste, I'interlocuteur faisait ainsi référence au fameux hebdomadaire, leadeur national du journalisme d'investigation et figure de proue de la presse critique mexicaine ${ }^{9}$. Cette question de la part des interlocuteurs reflétait leurs attentes vis-à-vis de la démarche de recherche: porter dans le débat public les problèmes auxquels ils font face et, de manière indirecte, contribuer à un changement social en leur faveur.

Ces deux exemples, qui nous conduisent à adopter des réactions très différentes, ne sont pas ici énoncés en vue de faire un étalage de telle ou telle expérience vécue par des chercheurs. Ils nous semblent valides au contraire pour dépasser les seuls récits individuels et rapprocher des situations vécues. II ne s'agit pas, dans ce cas, de mentionner le difficile accès au terrain : nous estimons en effet que tout travail de terrain présente ses difficultés et ses zones de danger.

En revanche, le caractère incertain, pour les interlocuteurs, des canaux de diffusion de l'information collectés localement, se traduit en effet de manières très différentes selon les lieux, les acteurs, et les contextes sociopolitiques dans lesquels ils sont inscrits : réticence et méfiance pour certains, espoir ou enthousiasme pour d'autres. Le malentendu sur les modalités de diffusion du savoir scientifique est à l'image du décalage existant entre les préoccupations quotidiennes des acteurs, marquées ici par l'urgence sociale face à la menace de perdre leur logement pour les uns, le risque d'être dévoilé voire dénoncé pour les autres, et nos impératifs scientifiques. Mais ce malentendu penche souvent en faveur du chercheur. Dans le meilleur des cas, nos

\footnotetext{
${ }^{9}$ Depuis 2007, les reporters de la revue analysent et dénoncent notamment de manière constante les dégâts sociaux liés à la guerre contre le narcotrafic menée par le gouvernement fédéral. Ce choix éditorial et politique a même conduit la rédaction de la revue à rendre anonyme certains articles pour préserver l'intégrité physique de leurs auteurs. Sur les questions les plus sensibles (corruption, narcotrafic), le propos étant assumé collectivement.
} 
articles seront publiés dans une revue hyperspécialisée, tirée à quelques centaines d'exemplaires et dont le lectorat se compose quasi exclusivement d'autres chercheurs dont les travaux s'inscrivent dans le même " micro sous-champ " de recherche (Chamayou, 2009). Publié, il deviendra une ligne de notre curriculum vitae mais n'aura, concrètement, aucune incidence dans la vie de ceux dont il traite. Mais est-ce seulement son rôle?

Ce questionnement sur l'utilité des savoirs est au cœur d'une réflexion sur l'éthique de la recherche qui transparait dans de nombreux travaux de géographes ${ }^{10}$, et témoigne des conflits internes que connaissent les chercheur-es, à titre individuel, quant à leur responsabilité vis-à-vis de leur terrain d'étude et des personnes ou groupes sociaux dont ils deviennent les porte-paroles à travers leurs publications. Étendue à l'ensemble de la communauté scientifique, il pose la question de l'utilité sociale de la recherche et du rapport entre les chercheur-es en sciences sociales et leurs objets d'étude, à plus forte raison lorsque les groupes sociaux étudiés subissent des formes de domination sociale. "Faut-il entrer dans la lutte auprès des dominés ? ", s'interroge B. Collignon (2010) : les chercheurs " construisent leurs carrières grâce à la collaboration des "cherchés", mais quels bénéfices ceux-ci en tirent-ils?" (p.71). Ainsi formulé, le problème est présenté comme étant situé dans une logique de don et de contredon entre le chercheur et ses informateurs : ces derniers procureraient aux chercheur-es les informations qui lui permettront ensuite de construire une carrière à l'intérieur du monde académique; aussi, celui-ci leur serait, d'une certaine manière, redevable. Selon cette position, l'exigence de réciprocité justifie alors l'engagement du chercheur aux côtés des "enquêtés ". Mais la réciprocité doit-elle nécessairement être dirigée vers les groupes sociaux constitués en sujets/objets de recherche ? On imagine mal, dans ce cas, le type de principes éthiques sur lesquels s'appuierait une recherche portant sur les catégories sociales dominantes. Si c'était le cas, les travaux de Monique et Michel Pinçon-Charlot sur la grande bourgeoisie auraient conduit leurs auteurs à un engagement aux côtés des puissants afin d'optimiser l'efficacité des outils de domination et de reproduction sociale. Si la possibilité d'un tel détournement de la sociologie critique est évoquée par les sociologues en annexe de leur ouvrage Les Ghettos du Gotha, leur projet scientifique n'en demeure pas moins de « mettre au jour des inégalités et des privilèges mal connus et d'en souligner les effets sociaux " (Pinçon et Pinçon Charlot, 2007, p.274). Dans ce cas, cela implique de s'engager non pas avec, mais contre leurs enquêtés ${ }^{11}$.

Cependant, la possibilité d'une subversion de la pensée critique pose par ailleurs les limites d'une recherche dont la seule portée serait de dévoiler les mécanismes économiques, politiques et sociaux à travers lesquels est perpétuée la domination. Les problèmes éthiques évoqués plus haut se présentent donc de manière d'autant plus aigüe aux chercheurs affichant la volonté d'articuler leur démarche scientifique à

\footnotetext{
${ }^{10}$ Outre les exemples citées à plusieurs reprises au cours de cet article (Morelle \& Ripoll, 2010 ; Alduy \& Labussière, à paraître) citons, parmi d'autres, la contribution de David Sibley, dans l'ouvrage collectif, Géographies anglo-saxonnes. Tendances contemporaines, Paris, Belin, 2001.

${ }^{11}$ Cette volonté de contribuer à la remise en cause d'un ordre social inégalitaire a été réaffirmée trois ans plus tard lors de la publication de leur dernier ouvrage, Le président des riches (2010) par un choix éditorial assurant la diffusion d'un savoir sociologique critique auprès d'un large public. La version numérique du livre est en effet en accès libre sur le site de l'éditeur, Zones.
} 
l'élaboration d'une critique sociale, et invitent à une réflexion sur les fondements mêmes d'une géographie critique.

\section{D’une géographie du dévoilement à une géographie "performative ": cheminements vers une recherche-action?}

Toute démarche de recherche soulève des questions d'éthique. Au-delà de la seule question du rapport aux enquêtés, ces interrogations permettent de penser à nouveaux frais la portée des recherches en sciences sociales. Quel pourrait-être ne serait-ce que le sens d'un article, d'une thèse, ou d'un ouvrage, aussi vifs soient-ils dans leurs propos, si la critique reste sans effet sur la réalité sociale elle-même ? Que reste-t-il d'une pensée lorsque celle-ci est déconnectée de l'action politique, sans prise possible sur le réel ? Ne risque-t-elle pas, comme le suggère P. Rimbert, d'être réduite à l'état de discours incantatoire menaçant "l'ordre des mots plus que celui des choses " (lbid.) ?

Ces questions doivent nous conduire à une prise de recul vis-à-vis de l'apparent renouveau de la pensée critique, illustré en géographie par un engouement pour une lecture radicale et contestataire notamment influencée par la géographie anglosaxonne, d'inspiration marxiste, et de sa normalisation autour d'auteurs tels que D. Harvey, E. Soja. Ce même recul est de mise pour envisager le retour actuel vers des figures importantes de la pensée critique française, comme H. Lefebvre (Garnier, 2011). Les récentes traductions en français de travaux anglo-saxons et, en de nombreuses langues, de philosophes et sociologues français, la multiplication de colloques et de numéros thématiques de revues scientifiques, en dépit de leurs succès éditoriaux et des tendances au rapprochement des savoirs contestataires qu'elles permettent, ne doivent cependant pas masquer le risque de laisser ce savoir " confiné aux donjons des citadelles académiques ", pour reprendre une formule empruntée à $P$. Rimbert.

L'engouement pour la pensée critique chez les chercheur-es en sciences sociales en France ou aux Etats-Unis par exemple a été très présent entre la fin des années 1960 et le début des années 1980 en géographie, notamment sous l'effet des tenants de la géographie radicale dans le monde anglo-saxon qui demeure en dépit d'une fragilisation qui a conduit à en annoncer "la mort " (Buttimer, 1999, p.103, citée par Collignon, 2001, p.136), et de la géographie sociale en France. Le mouvement récent observé en France est aujourd'hui à considérer avec d'autant plus de prudence, voire de circonspection, qu'il intervient à un moment de rupture dans le fonctionnement du monde scientifique. Face à la généralisation de la recherche sur contrats, aux modes restrictifs et comptables d'évaluation de la production et à l'autonomie des établissements, les universitaires sont toujours plus nombreux dans le monde à être confrontés à leur propre incapacité à peser collectivement et durablement sur l'évolution des cadres de la production des savoirs académiques. Or c'est à l'intérieur même de ces cadres que prend place l'actuelle réflexion sur la pensée critique.

Selon, R. Keucheyan, les (nouvelles) théories critiques auraient pour principal dénominateur commun le fait qu'elles « remettent en question l'ordre social existant de façon globale " $(2011$, p.2). Si cette définition doit bien entendu porter sur leur contenu même, elle peut difficilement faire l'économie d'une réflexion sur l'efficacité de ces théories, envisagées comme des instruments au service du changement social. 
L'entreprise est difficile, comme le souligne très justement le géographie britannique D. Sibley, lorsqu'il écrit que «le contexte disciplinaire dans lequel la plupart des géographes travaillent (...) paraît inhibant, car il les porte à pratiquer eux-mêmes des exclusions. Sans vouloir paraitre trop directif, il me semble que si l'on souhaite que la géographie rende compte de la différence de façon authentique et combatte les tendances à l'exclusion, il convient que ses praticiens transgressent les barrières disciplinaires et interpersonnelles et deviennent beaucoup plus proches des gens dont les problèmes donnent à la géographie sa première raison d'exister ", (Sibley, 2001, p.37).

Ces deux facettes de la pensée critique sont présentes dans la définition du "savoir engagé " proposée par P. Bourdieu. Aux fonctions dites " négatives ", de critique de ce qui est, doit être articulée une fonction "positive " correspondant à " un travail collectif d'invention politique " (Bourdieu, 2001). En d'autres termes, il s'agit de passer d'une " sociologie du dévoilement ", visant à rendre intelligibles les ressorts de l'ordre social mis en cause, à une sociologie "performative ", participant à la construction collective d'une réalité sociale autre (Callon, 1999, p.72). Le fait de transposer cette réflexion à notre champ disciplinaire permet d'envisager une géographie sociale qui, plusieurs décennies après les préceptes de R. Rochefort, doit " prendre ses risques, s'efforcer non plus seulement d'enregistrer des résultats, mais de s'insinuer dans la genèse des choses et de suivre les cheminements multiples d'un monde en voie d'élaboration " (Rochefort, 1962, p.2). Cette invitation, pourtant relayée par des générations de géographes, ne semble pas avoir permis l'établissement d'un débat de fond dans la discipline. Nous ne nous risquerons pas à supposer que le contexte actuel qui prévaut à la production scientifique conduirait en quelque sorte à limiter la prise de risque ou la production d'alternative en même temps qu'il contribuerait à affirmer le confinement de la portée critique du discours scientifique. Nul doute par ailleurs que les raisons de ce phénomène dépassent largement le seul domaine de la recherche.

Reste qu'il s'agit de ne pas minimiser les effets potentiels du travail de " dévoilement » qui incombe à toute critique. Celui-ci constitue l'un des ressorts majeurs de la pensée critique lorsque son projet est de " rendre la réalité inacceptable et, par là, d'engager les personnes auxquelles [elle s'adresse] dans des actions qui doivent avoir pour résultat d'en changer les contours " (Boltanski, 2009, p.21). La transformation de la réalité est alors étroitement liée à la réception du travail scientifique. Dévoiler, mais à qui ? Les écrits scientifiques " critiques » ne sont-ils pas hors de portée des catégories sociales dont nous traitons parfois et qui sont celles qui auraient le plus intérêt à ce que la réalité soit transformée?

Ce questionnement traverse de manière récurrente les géographies critiques issues de différents contextes sociétaux et académiques. Le formuler à nouveau n'a de sens que si ce constat constitue le premier jalon d'un projet scientifique (et politique) ayant pour but de peser sur la marche des sociétés, projet dont l'urgence et la difficulté ont été signalées par de nombreux auteurs (Bourdieu, 2001 ; Kayser, 1978). C'est ainsi, il nous semble, qu'il faut entendre le propos de D. Harvey lorsqu'il déclarait, en 1984, que " la géographie est une chose trop importante pour être laissée aux géographes » (Harvey, 2010, p.46). En France, cette même inquiétude anime notamment le projet éditorial de la revue Hérodote, comme le soulignait à la fin des années 1970 l'éditorial d'un numéro consacré à l'enquête de terrain (1978): " II nous parait essentiel que le 
savoir-penser-l'espace qui est actuellement - et dans tous les régimes - l'apanage des minorités dirigeantes (...) puisse devenir, tout comme le savoir-lire-et-écrire, l'outillage intellectuel de tous les citoyens" (p.5). À l'évidence, et c'est toujours le cas aujourd'hui, la portée des publications scientifiques semble dérisoire face à la force de frappe des dispositifs médiatiques relayant les discours dominants.

À l'inverse, on peut aller jusqu'à imaginer des situations dans lesquelles le savoir critique sert finalement les intérêts de classes qu'il prétend remettre en cause, comme en témoigne l'expérience de $\mathrm{M}$. Pinçon et $\mathrm{M}$. Pinçon-Charlot : " en général, nos textes sont bien acceptés par le milieu fortuné de la noblesse et de la grande bourgeoisie ancienne. Ce qui nous étonne d'autant plus que nous utilisons le système théorique élaboré par Pierre Bourdieu. (...) Nos livres, agrémentés du label scientifique que nous procure l'appartenance au CNRS, leur ouvrent de nouveaux espaces de compréhension de leurs propres pratiques. Bien plus, ils peuvent instrumentaliser nos analyses des processus de la reproduction sociale pour affiner leurs stratégies. Une sociologie critique peut devenir une arme pour la défense de positions dominantes dont elle dévoile le fonctionnement » (2007, p.275).

Pour qu'une recherche puisse être considérée comme critique, elle doit donc nécessairement intégrer un effort de réflexivité critique sur la portée sociale et politique des savoirs produits. La responsabilité des chercheurs s'en trouve engagée à chaque étape du travail scientifique, de la construction de l'objet à la réception du rendu final. La portée critique d'une recherche en sciences sociales réside donc autant dans son contenu même que dans la relation créée tout au long de ce processus entre le chercheur, son objet et les destinataires du savoir produit. Cette idée a notamment été développée par L. Boltanski, pour qui «l'idée d'une théorie critique qui ne serait pas adossée à l'expérience d'un collectif et qui existerait en quelque sorte pour ellemême, c'est-à-dire pour personne, est inconsistante » (2009, p.21).

Le caractère opératoire des savoirs géographiques "critiques" dépend de leur appropriation par les acteurs susceptibles de s'en saisir pour agir sur le réel. Cela implique, pour les chercheurs, de "transcender la frontière sacrée (...) entre le scholarship et le commitment, pour sortir résolument du microcosme académique, entrer en interaction avec le monde extérieur " (Bourdieu, 2001). Cette nécessité est aujourd'hui réaffirmée, tandis que les nouvelles logiques de publication de la recherche scientifique incitent les chercheurs à orienter la publication de leurs travaux vers des espaces de diffusion toujours plus éloignés du grand public (revues indexées, poids de l'impact factor).

Au-delà des débats désormais anciens autour de couples conceptuels tels que science et action, objectivité et subjectivité, l'actualité de la réflexion sur l'engagement des chercheurs ne saurait être saisie pleinement sans prendre en compte les mutations contemporaines internes au champ académique et l'alignement de celui-ci sur les logiques de marché inhérentes au monde économique. Nous rejoignons sur ce point le sociologue R. Chartier pour qui «la relation entre les structures des productions intellectuelles et esthétiques et l'identité sociale de leurs producteurs [est] toujours médiatisée par les lois spécifiques qui gouvernent les hiérarchies et polarisations du champ qui est le leur »(2003, p.255). Les apports de la sociologie bourdieusienne quant à la compréhension du champ culturel et de "l'économie des biens symboliques " s'avèrent en effet particulièrement utiles pour interroger les conditions de production du discours scientifique critique et, plus spécifiquement, celle de la 
publication des travaux scientifiques. Car l'utilité sociale du savoir universitaire apparait être un horizon bien improbable, alors que les nouvelles normes de publication deviennent l'un des principaux critères d'évaluation de la recherche et déterminent des aspects aussi fondamentaux de l'activité scientifique que le montant des dotations financières attribuées aux laboratoires, mais aussi l'accès à l'emploi pour des chercheurs précaires soumis à une concurrence exacerbée. Si les positions et les trajectoires sociales des chercheurs, et notamment la convergence de leurs intérêts de classe avec ceux des catégories "dominantes " ${ }^{12}$ sont parfois invoquées pour expliquer la déconnexion entre champ académique et action politique, celle-ci doit aussi être considérée au regard de la précarisation croissante des personnels universitaires et de sa traduction en termes d'aliénation, tant sur le plan matériel que sur celui de la production scientifique ${ }^{13}$.

Sur la base de ces constats, relayant - pour partie - la réflexion proposée par Alduy et Labussière ( $/ \mathrm{bid}$.) et à la lumière de questionnements issus de notre pratique de la recherche, nous souhaitons envisager les formes que pourraient prendre une recherche-action critique.

\section{Pour une géographie critique de l'action}

L'inventivité des chercheurs dans la création de liens avec la société, d'outils de diffusion des savoirs et d'invention politique pourrait bien apparaitre comme le creuset d'une radicalité renouvelée, concrète, nécessairement articulée à un questionnement rigoureux sur les cadres théoriques mobilisés. Cela passe notamment par un renforcement des relations entre chercheurs et non-chercheurs, entre les équipes de recherche et leur environnement social, tant dans le cadre des recherches de "terrain " qu'à travers les travaux d'enseignement et de diffusion scientifique ${ }^{14}$. Mais la tâche est d'autant plus difficile et ambigüe que les choix qu'elle implique sont autant d'ordre politique que théorique. Car la " société » à laquelle il conviendrait de s'associer, si localisée soit-elle, n'est jamais une totalité exempte de contradictions, de rapports de force, d'intérêts parfois irréconciliables. Comme le signale M. Callon, s'associer avec des acteurs, "c'est retirer des marges de manœuvre à d'autres acteurs, c'est contribuer à fabriquer des asymétries et à rendre impossibles certaines configurations. Il s'agit donc d'un travail politique " (Callon, 1999).

Cette volonté de décloisonner les savoirs académiques et d'ancrer le discours scientifique dans la pratique sociale traverse des travaux ayant marqué la géographie sociale française. Dans sa thèse sur le travail en Sicile, R. Rochefort assume ainsi une posture l'amenant à devenir, en quelque sorte, porte-parole des réalités sociales rencontrées sur son terrain (Labussière, 2008) : " le langage géographique est d'abord un langage concret. La peine des hommes se décrit : elle s'évoque, elle se raconte » (Rochefort, 1961, citée par Labussière). En prise avec le terrain, affichant la volonté de

\footnotetext{
12 Nous faisons ici référence à l'usage de la catégorie " petite bourgeoisie intellectuelle (PBI) » dans l'analyse développée par Jean-Pierre Garnier

${ }^{13}$ L'étude la plus récente sur ce sujet dans le contexte français est sans doute l'ouvrage " Recherche atomisée, recherche précarisée " (P.E.C.R.E.S., 2011), paru aux éditions Raisons d'Agir.

${ }^{14}$ Ce terme nous semble plus approprié que celui de " vulgarisation », qui, selon L. Zomboni, est " sujet à l'éventuelle critique de porter en soi une connotation négative » (2001, p.49).
} 
produire un discours accessible au plus grand nombre, la géographie sociale ne renonce pas pour autant à la production théorique. Elle est même, selon R. Hérin, " théorique par nécessité » (entretien, 10 février 2012). En produisant de la généralité, le chercheur favorise ainsi l'intelligibilité, la compréhension des processus locaux et de leur inscription dans des logiques globales. Pour M. Callon, ce rapport entre théorie et pratique est une dimension centrale du travail sociologique : "le seul apport des sciences sociales, mais il est immense, est de participer avec les acteurs eux-mêmes à la mise en forme des leçons qui peuvent être tirées d'une expérience collective en cours, toujours singulière, de manière à en exprimer la possible généralité pour ensuite la transporter ailleurs, en espérant que d'autres acteurs seront convaincus par l'équivalence et s'en saisiront " (Callon, 1999, p.74). Cet effort de généralisation ne doit pas être limité à la production du rendu final de la recherche, mais traverser ses différents moments, dont l'enquête de terrain. "N'est-il pas vrai qu'à travers les contacts et les discussions qui s'instaurent au cours des enquêtes le chercheur peut être directement utile à ses interlocuteurs, leur communiquant des informations, les aidant à interpréter les pressions ou les interventions dont ils font l'objet, à replacer leurs problèmes spécifiques dans la problématique générale ? ", insiste B. Kayser dans un fameux texte consacré au rapport des géographes à leurs terrains de recherche (Kayser, 1978, p.10). Pour R. Hérin, le rôle du géographe peut alors être celui d'un "transmetteur", d'un " animateur ", la production de la connaissance devenant un travail collectif, résolument tourné vers la société et la résolution des problèmes sociaux. Le projet de la géographie sociale rejoint là celui de la sociologie critique, énoncé par P. Bourdieu, pour qui le chercheur constitue une sorte $d$ ' " accoucheur " (Bourdieu, 2001), jouant un rôle actif dans la transformation du social.

C'est dans cet esprit que s'inscrivent de nombreuses initiatives qui, en dépit d'émerger dans des contextes sociogéographiques différents, partagent la même ambition de rapprocher des expériences et des savoirs et, suivant la proposition formulée par D. Sibley de rapprocher chercheur-es et enquêté-es. Plusieurs exemples permettent d'entrevoir les éventuelles perspectives qu'elles ouvrent, de même que les difficultés qu'elles éprouvent à envisager la production d'un savoir critique inscrit dans l'action.

En 2008, au Mexique, un groupe de doctorants et de chercheurs du département d'anthropologie de l'Université Autonome Métropolitaine (UAM) de Mexico a mis en place une expérience de coproduction des savoirs géographiques sur les formes d'autonomie développées par des organisations sociales indigènes de différentes régions mexicaines (Gasparello, Quintana Guerrero, 2009). Cette réflexion prend un sens particulier dans une société marquée par 70 années d'hégémonie d'un parti politique, le Parti Révolutionnaire Institutionnel (PRI), et par son emprise sur les différentes strates de la société (organisations ouvrières, paysannes, communautés agraires, assemblées de quartiers, etc.). Pour les organisations sociales d'opposition, et plus spécifiquement pour les organisations paysannes, la remise en cause des liens organiques avec les partis politiques et avec l'État constitue depuis plusieurs décennies un champ d'innovation sociale et politique autour de formes d'organisations sociales et territoriales plaçant l'autonomie au cœur du projet d'émancipation. Pour ces mouvements, la pensée théorique sur la question de l'autonomie (et notamment les travaux de C. Castoriadis) a été un instrument pour l'action politique, tandis que les expériences locales d'organisation autonomes, à l'image du projet zapatiste, ont nourri 
la réflexion scientifique et alimenté un grand nombre d'échanges entre chercheurs et non-chercheurs, entre le monde académique et les mouvements sociaux.

Dans cette optique, une table ronde réunissant chercheurs, journalistes engagés et représentants de diverses organisations Indigènes s'est tenue à la UAM en 2008 dans le cadre d'un débat sur la question de l'autonomie. Une fois retranscrits, les interventions et les échanges entre les participants ont été retournés à leurs auteurs respectifs afin d'être repris et mis en forme en vue d'une publication collective. Le résultat final de ces rencontres est un objet scientifique hybride dans lequel s'entremêlent et se répondent récits d'expériences et retours critiques sur les processus et les formes organisationnelles. La parole scientifique nourrit ainsi l'entreprise réflexive et devient une courroie de transmission entre les expériences locales, contribuant à dessiner ces " autres géographies " ("Otras Geografias »), celles que produisent, par l'action collective, les organisations indigènes autonomes.

Dans le même registre, ailleurs, on peut mentionner le projet de "géographie populaire ${ }^{15}$ impulsé dans l'Etat de New York, par Don Mitchell ${ }^{16}$, figure importante de la géographie radicale anglo-saxonne, avec l'ambition affichée de " populariser la géographie radicale ou radicaliser la géographie populaire " (Tonnelat, 2009). Face au confinement des savoirs géographiques radicaux aux seuls milieux universitaires et journalistiques (cet "enclos universitaire» dénoncé par P. Rimbert), l'une des réponses du projet de géographie populaire a été de réfléchir aux canaux de diffusion des savoirs: production de brochures, de vidéos, d'ouvrages dits de "vulgarisation ", mais aussi cartographie de la pauvreté dans la ville, etc. "Nos projets partent de la base ", affirme en effet D. Mitchell. " Une de mes tâches (...) c'est, alors que beaucoup de ces choses sont terriblement pratiques, d'amener les gens à réfléchir en quoi elles reflètent des forces sociales plus larges » (Tonnelat, 2009, p.7). Cet effort passe par un rapprochement entre chercheur-es et militant-es (en invitant notamment ces derniers au Congrès National de Géographie, I'Université prenant en charge leurs frais d'inscription), et en particulier avec les coalitions pour le droit à la ville présentes dans différentes villes américaines.

Ce rapprochement entre sphères académiques et militantes est observé dans d'autres contextes, certains dans lesquels nous avons travaillé. II nous semble particulièrement intéressant à noter dans le contexte de Mumbai, où plusieurs travaux sur les conséquences sociales des politiques de réhabilitation de bidonvilles (Slum Rehabilitation Schemes) - notamment les expulsions forcées - ont donné lieu à des interventions publiques et à des publications coécrites par des membres d'Organisations Non Gouvernementales et des chercheur-es en sciences humaines et sociales ${ }^{17}$. Cette pratique de publication est peu habituelle en France, où le monde académique voit d'un œil suspicieux toute forme de rapprochement institutionnel, par ailleurs ici tout à fait explicite, alors même que les chercheur-es ayant recours à des formes de productions semblables sans pour autant les expliciter sont nombreux, l'exemple des rapports de recherche et des études établis dans le cadre de commandes émanant de collectivités et engageant étudiants en formation

\footnotetext{
${ }^{15}$ Le titre du projet fait directement référence à l'ouvrage de l'historien $\mathrm{H}$. Zinn, Une histoire populaire des États-Unis.

${ }^{16}$ http://www.peoplesgeographyproject.org/

${ }^{17}$ Voir notamment Patel, Sheela, D’Cruz Celine, Burra Sundar (2002) “Beyond Evictions in a Global City: People-Managed Resettlement in Mumbai." Environment and Urbanization 14(1).
} 
professionnalisante et enseignants est, à ce titre, éloquent. Hors du contexte français, au sein duquel de telles collaborations mériteraient d'ailleurs d'être éclairées tant elles nous paraissent constituer un domaine d'intervention encore sous-investi par le champ de la critique académique, une recherche participative à laquelle nous avons contribué à Mumbai nous semble intéressante à explorer plus avant. Inscrite dans une ambition assez proche de celles déjà mentionnées, mais sans affiliation universitaire directe, le travail conduit depuis plusieurs années dans le cadre de l'ONG PUKAR (Partner for Urban Knowledge and Action Research) propose un travail d'enquête au plus près des résidants de plusieurs quartiers de la ville, sollicitant leurs points de vue, les associant à la démarche d'entretiens, et diffusant les résultats dans le cadre de réunions publiques. Selon cette organisation, les groupes de résidants doivent jouer un rôle actif dans la définition, la mise en place et la maintenance des programmes de développement et des politiques locales. Pour encadrer et systématiser cette pratique, PUKAR a mis en place, depuis 2005, des bourses de recherche destinées à des " barefoot scholars " (littéralement "des chercheurs aux pieds nus »), visant à donner une chance à des personnes situées hors du champ académique de prendre part à la production scientifique, moyennant une rétribution sous forme salariale.

Conscients que les conceptions et les dispositifs afférents à ce type de démarche, désignés par les expressions de "planification participative ", de "gouvernance collaborative ", ou encore $d$ ' " empowered publics ${ }^{18}$, sont susceptibles d'être récupérés et utilisés pour "veil the nature and effects of power and...hold out the prospects of democracy without the inconveniences of contestational politics and of the conflicts of ideas and interests that are an essential part of democracy" (Harriss 2001, 118), leurs instigateurs considèrent néanmoins qu'il est important d'élargir à la fois les producteurs et l'audience de la recherche en renforçant des "communautés discursives". Celles-ci sont, selon l'auteur, les seules instances en mesure de constituer des contrepouvoirs aux pouvoirs dominants, au travers de coalitions non pas engagées de manière frontale vis-à-vis des pouvoirs gouvernementaux dominants-, mais selon une approche collaborative ${ }^{19}$.

Les références à ces initiatives nous semblent offrir des perspectives pour envisager d'un regard nouveau de nouvelles formes de recherche-action, en ce qu'elles s'essayent à concilier deux aspects qui nous semblent, dans la recherche, trop peu souvent pensés ensemble : dévoiler des structures de compréhension des sociétés (ou de leurs relations à l'espace) en invitant celles-ci à s'en emparer, tout en essayant de limiter la distance entre les productions scientifiques et le reste de la société. C'est dans cet esprit que fût notamment créée, autour de Robert Hérin et de plusieurs collègues de l'Université de Caen, une "université des quartiers populaires ". Dans l'esprit de ses initiateurs, ce projet se voulait une sorte d'envers de l'université dite " populaire " proposée par le philosophe Michel Onfray, destinée à la bourgeoisie intellectualisante. II se fondait sur un rapprochement entre chercheur-es et résidants de quartiers de la ville de Caen, sans porte-parole, dans un projet d'échanges et de co-

\footnotetext{
18 II n'existe pas, à notre connaissance de traduction française correcte du mot " empowerment ". Le terme renvoie à l'idée de renforcement du pouvoir de la population, sous-entendu des catégories sociales en difficulté.

${ }^{19}$ Cette expression renvoie à la réflexion menée par P. Healey, 1997. Collaborative planning. Shaping places in fragmented societies, Londres: Mac Millan.
} 
construction de savoirs. Comme d'autres avant et après lui, ce projet n'aura duré que quelques mois, fragilisé par des préoccupations souvent peu conciliables entre scientifiques et citoyens.

Entre les expériences évoquées sur nos terrains de recherche respectifs (Amérique latine, Inde) et celles qui, par le passé, ont vu l'émergence de mouvements universitaires populaires à l'exemple de celle mentionnée ici, les contextes sociopolitiques diffèrent certes très largement. Nul doute néanmoins que demeure un univers des possibles pour inscrire la critique dans le champ de l'action, qu'elle soit explicitement sociale ou, plus largement, inscrite dans le contexte de l'action " territoriale ».

La portée critique de la production scientifique ne réside ainsi pas seulement dans le rendu écrit. Elle doit selon nous être contenue plus largement dans le processus de production de la connaissance et dans ses tentatives d'articulation avec l'action collective et avec la société. Certes, la position idéale du chercheur, à la fois intellectuel productif responsable scientifiquement - transmetteur en quelque sorte et animateur semble difficile d'accès. Cette quête est trop souvent obstruée par la prétention d'être porteur d'une responsabilité politique : or, le fait d'être responsable scientifiquement signifie être dans le devoir de diffuser les connaissances au public le plus large possible, et pas nécessairement à être porteur de responsabilité ou de pouvoir politique.

\section{Bibliographie}

Bautès N., Boissinot E. et Saglio-Yatzimirsky M-C. (2011) "Ressources foncières et pression immobilière à Mumbai " Revue Tiers Monde, Dynamiques foncières dans les villes du Sud, $n^{\circ} 206$, pp. 55-74.

Boltanski L. (2009), De la critique. Précis de sociologie de l'émancipation, Paris, Gallimard, $275 \mathrm{p}$.

Bourdieu P. (2001), Contre-Feux 2, Paris, Raisons d'agir, 128 p.

Callon M. (1999) « Ni intellectuel engagé, ni intellectuel dégagé : la double stratégie de l'attachement et du détachement ", Sociologie du travail, 1999/41, pp. 65-78.

Chamayou G. (2009) « Petits conseils aux enseignants chercheurs qui voudront réussir leur évaluation ", Contretemps, http://www.contretemps.eu/interventions/petitsconseils-enseignants-chercheurs-qui-voudront-reussir-leur-evaluation

Collignon B. (2010) "L'éthique et le terrain », L'information Géographique, 2010/74, pp. 63-84.

Collignon B. (2001) " La géographie radicale : à la recherche d'un nouveau souffle " in : Staszak J.-F. (dir.), Géographies anglo-saxonnes. Tendances contemporaines, Paris, Belin, pp. 131-138.

Frémont A., Hérin R., Chevalier J. et Renard J. (1984), Géographie sociale, Masson, 387 p.

Garnier J-P. (2011) "Voies et moyens pour le retour d'une pensée critique " radicale » de l'urbain ", Article11, http://www.article11.info/?Voies-et-moyens-pour-le-retour-d Gasparello Q. G. (2009), Otras Geografías. Experiencias de autonomías indígenas en México, Mexico, Universidad Autónoma Metropolitana, 233 p.

Ghasarian C., (1997) « Les désarrois de l'ethnographe », L'Homme, 143, pp. 189-198. 
Hancock C. (2007) " "Délivrez-nous de l'exotisme ": quelques réflexions sur des impensés de la recherche géographique sur les Suds (et les Nords) ", Autrepart, 2007/1 $\mathrm{n}^{\circ} 41$, p. 69-81.

Harriss J. (2001), Depoliticizing Development : The World Bank and Social Capital, Londres,

Anthem Press, $158 \mathrm{p}$.

Harvey D. (2010), Géographie et Capital, Paris, Syllepses, 280 p.

Keucheyan R. (2010) Hémisphère gauche. Une cartographie des nouvelles pensées critiques, Paris, Zones, 316 p. (En ligne: http://www.editionszones.fr/spip.php?page=lyberplayer\&id article=108)

Labussière O. et Aldhuy J. (en cours d'évaluation) (2011) " Le terrain? C'est ce qui résiste. Réflexion sur la dimension cognitive de l'expérience sensible en géographie ", Annales de Géographie (En ligne: hal.archivesouvertes.fr/.../Labussiere_Aldhuy_Le_terrain_resiste.pdf, page consultée le 15 octobre 2011.

Omasombo-Tshonda J. dir. (1993), Le Zaïre à l'épreuve de I'histoire immédiate: hommage à Benoît Verhaegen, Paris, Karthala, Coll. Hommes et Sociétés, 310 p.

Martiniello M. et Simon P. (2005) "Les enjeux de la catégorisation. Rapports de domination et luttes autour de la représentation dans les sociétés post-migratoires " Revue européenne des migrations internationales, 21/2, pp.7-18.

Morelle M. et Ripoll F. (2009) "Les chercheur-es face aux injustices : l'enquête de terrain comme épreuve éthique ", Annales de géographie, 2009/665-666, pp. 157-168. Pinçon M. et Pinçon-Charlot M. (2007), Les Ghettos du Gotha. Comment la bourgeoisie défend ses espaces, Paris, Le Seuil, 295 p.

Racine J-B. (1989) "Le métier de géographe : le recours aux sources. Commentaires àpropos d'une pensée nouvelle au sein de la géographie française ", Cahiers de géographie du Québec, vol. 33, n 88, p. 51-57.

Rimbert P. (2011) "La pensée critique dans l'enclos universitaire ", Le Monde Diplomatique, janvier.

Rochefort R. (1983) "Réflexions liminaires sur la géographie sociale" in Noin D. (dir.), Actes du Colloque de Lyon, 14-16 oct. 1982, Paris, Centre de Polycopie de I'Université de Paris 1, pp. 11-15.

Rochefort R. (1961), Le travail en Sicile. Etude de géographie sociale. Presses Universitaires de France, $363 \mathrm{p}$.

Soudière (de La) M. (1988) " "Faire " la Creuse, le Maroc, la Lozère... (A propos des ouvrages Ethnologue au Maroc, réflexion sur une enquête de terrain de Paul Rabinow, et Vivre dans la Creuse de Jacques Maho)", Terrain, 11, Mélanges. http://www.terrain.revues.org/3316.

Tonnelat S. (2009), "Un entretien avec Don Mitchell », Justice Spatiale Spatial Justice, $\mathrm{n}^{\circ} 1$.

Vant A. (1984) "La géographie sociale lyonnaise en perspective ", Revue de Géographie de Lyon, 1984/3, pp. 131-143. 